\title{
La gestión de la información en un enfoque a partir de la entropía
}

\section{The information management in an entropy approach}

\section{Gestión de información y entropía}

\author{
Rafael M. Ávila Ávila. $\mathrm{PhD}^{(1)}$ \\ Julio César Pino Tarragó. $\mathrm{PhD}^{(2)}$ \\ María del Carmen Expósito Gallardo. M.Sc. ${ }^{(3)}$ \\ Ing. Dunia Lisbet Domínguez Gálvez. Ing. ${ }^{(4)}$
}

(1) Universidad de Holguín. Cuba, ravilaa@uho.uho.edu.cu

(2) Universidad Estatal del Sur de Manabí. Ecuador, jpinoecuador@gmail.com

(3) Universidad Médica Mariana Grajales Coello, Cuba, mariaex@infomed.sld.cu

(4) Universidad Estatal del Sur de Manabí. Ecuador, juliodunia1406@ gmail.com

Contacto: ravilaa@uho.edu.cu

\section{Resumen:}

La entropía y la información están íntimamente relacionadas. A partir de una revisión de la teoría matemática que involucra a estos conceptos, los objetivos se centran en sintetizar y analizar los elementos teóricos básicos así como valorar el concepto de entropía asociado al manejo informacional y sus implicaciones en la organización de una entidad. En tal contexto, se discuten las propiedades fundamentales de la entropía desde el punto de vista termodinámicos y fuera de éste marco, así como los rasgos de la información que pueden conducir a un redimensionamiento de su gestión teniendo en cuenta los factores estocásticos que propician efectos de desorganización sobre el sistema. Se concluye que dicha magnitud representa un recurso metodológico para enfocar la gestión de información en el proceso de funcionamiento de las entidades como un sistema.

Palabras claves: información, gestión, entropía, organización, empresa.

\footnotetext{
Abstract:

The entropy and information are related. From the review of the mathematical theory involving these concepts, the objective are to analyze and synthesize the basic elements as well as to value the concept of entropy associated to the information management and its implications in the enterprise organization. In such context, the fundamental properties on entropy are discussed from the thermodynamic point of view and out of this frame as well as the features of information, which can give rise to a redimensioning of its management, taking into account stochastic factors
}

that can produce disorders in the system. It is concluded that such magnitude represents a methodological resource to focus the information management in the process of functioning of the enterprise as a system.

Keywords: information, management, entropy, organization, enterprise.

\section{Introducción}

La información constituye un vocablo polisemántico, si bien ya Norbert Wiener al referirse a él subrayó que no era ni energía ni materia (Wiener, 1965). Algunos autores la definen como "característica de la organización interior del sistema material, conforme a los numerosos estados que la misma puede adquirir" (Kolmogorov, V.M.Glushkov y W.R. Ashby) y que permite estimar las posibilidades potenciales de dichos sistemas, con independencia del proceso de transmisión o reproducción de la misma (Dimitriev, 1991).

La función informacional se refiere a la capacidad de recepción, reelaboración, generación y emisión de información. Dicha recepción conduce a la eliminación o reducción de incertidumbres o indeterminaciones (Mihail y Mircea, 1989). En consecuencia, sin la información es difícil concebir un sistema ordenado pues esta le confiere seguridad a la organización del mismo; de ahí la necesidad de la gestión de información a partir de un conjunto de actividades encaminadas al control, el almacenamiento y la posterior recuperación de aquella que es producida, recibida o retenida durante el desarrollo de las actividades de cualquier organización (Bustelo y Amarilla, 2001). 
Los conceptos de información y entropía exhiben una profunda relación y resultan de gran utilidad la investigación de los sistemas de carácter bastante general, aunque la concepción de entropía procede de la Física y se asocia a situaciones de desorden, caos o falta de organización. Por su parte, la falta de disponibilidad de información en lo referido a la cantidad y calidad necesaria, genera comportamientos aleatorios en los sistemas y es caracterizada por la entropía informacional (Castell, 2000).

Una entidad de la sociedad como puede ser una organización empresarial ante las condiciones de progresiva informatización, cuya estructura no se corresponda con este proceso y con bajos niveles de gestión de información puede transitar hacia un sistema complejo estocástico, con dependencias acentuadas de los efectos entrópicos y baja eficiencia en el empleo de los recursos informativos. Ello a su vez genera un crecimiento de la demanda de información cuya satisfacción redundará en la transición a un estado de menor entropía, más ordenado (Прангишвили, 2003).

El enfoque basado en la entropía constituye una alternativa en el tratamiento de la gestión de la información. Su fundamento teórico metodológico contempla la revisión del concepto de entropía en su vínculo con el de información y su aplicación a sistemas de naturaleza no física, pues al parecer juega el papel de parámetro universal que permite dar solución a ciertos problemas referidos a los sistemas estocásticos complejos.

La descripción del comportamiento de los sistemas referidos basada en la noción de entropía, entraña algunas dificultades. Sin embargo ello no ha sido obstáculo para abordar el problema de la demanda de información teniendo en cuenta las incertidumbres, los mecanismos de balance entrópico y los modelos de procesos que tienen en cuenta medidas de indeterminación basadas en la entropía (Jae-Yoon, Chang-Ho y Cardoso, 2011).

La noción de entropía se ha tomado como base para cuantificar el impacto de la pérdida de información (Schwarz, 2014) [9] así como para la evaluación del grado de orden de los sistemas de información (Song, Liang y Ni). [10]. No resulta sorpresivo que varias publicaciones hayan tratado el enfoque entrópico y las aplicaciones de éste en la gestión de sistemas técnicos, biológicos, mecánicos, sociales, económicos aunque aún no es suficiente en el contexto de las investigaciones autóctonas, especialmente en lo referido a la gestión de la información.

A partir de lo establecido por la teoría matemática de la información y sus vínculos con la mecánica estadística, se abordan las propiedades de la entropía que sirven como base para el desarrollo de un enfoque de la gestión informacional basado en tal concepto. El objetivo principal consiste en valorar de manera general aquellas cuestiones que pueden resultar de interés para dicha gestión en el caso de diversas entidades así como las implicaciones en la concepción de la organización sistémica, teniendo en cuenta el modelo de Markina-Dyachkov (Markina y Dyachkov, 2014).

\section{Materiales y métodos}

En aras de localizar los documentos bibliográficos se emplearon varias fuentes documentales.

La búsqueda fue realizada en las bases de datos disponibles en los centros universitarios, bibliotecas y empresas accesibles, mediante el empleo de diferentes descriptores tales como entropía, gestión de información, análisis crítico, desorden empresarial, tanto en Idioma Español como en Inglés. También se realizó una La búsqueda en internet fue posible mediante palabras claves en los idiomas referidos además del Ruso y con ayuda del google académico.

Los documentos se seleccionaron teniendo en cuenta aspectos trascendentes referidos a la gestión de información en el ámbito empresarial en asociación con el concepto de entropía.

\section{Resultados}

\section{Fundamentos físico-matemáticos de la teoría de} la información

La cantidad de información y la entropía constituyen dos conceptos fundamentales de la teoría clásica que los sistematiza. La primera medida cuantitativa de la información fue dada por R. Hartley quien se basó en un modelo en el que se atribuían iguales probabilidades a todos los estados posibles de la fuente (Hartley, 1928). [11]. Sin embargo, la expresión más general para la cantidad de información y que corresponde a una sucesión de eventos que tienen diferentes valores de probabilidades de realización, fue planteada por C. Shannon (Shannon, 1948):

$$
H=-K \sum_{m=1}^{n} p_{i} \ln \left(p_{i}\right)
$$

$\mathrm{K}$ depende de las unidades de medición. Si la información es expresada en bits, $\mathrm{K}=(\ln 2)^{-1}$. Si K se iguala a la constante de Boltzmann $\mathrm{k}=1.38 \times 10^{-}$ ${ }^{23} \mathrm{~J} / \mathrm{K}$, la expresión (1) coincide con la Fórmula de Boltzmann para la entropía, de gran utilidad en el 
estudio de los sistemas que aborda la Mecánica Estadística:

$$
\mathrm{S}=-\mathrm{k} \sum_{\mathrm{m}=1}^{\mathrm{n}} \mathrm{p}_{\mathrm{i}} \ln \left(\mathrm{p}_{\mathrm{i}}\right)
$$

$\mathrm{H}$ se vincula también con la magnitud que aparece en el famoso Teorema-H de Boltzmann (Terlietski, 1971).

La medida dada por fórmula de Shannon (1) se refiere a la indeterminación en la elección de uno de los estados entre el conjunto de estados posibles, por parte de una fuente discreta. La coincidencia de su estructura formal con la estructura de la fórmula establecida por L. Boltzmann (2), ha hecho que a $\mathrm{H}$ también se le denomine entropía de una fuente discreta o del conjunto finito [3]. Tal coincidencia no es casual y tiene un profundo significado físico asociado a que tanto la entropía $\mathrm{S}$ como la cantidad de información $\mathrm{H}$ (ó I), constituyen una característica relevante del grado de diversidad del sistema.

Por otra parte, si se produce una transición en la distribución de probabilidades de $\mathrm{P}$ a $\mathrm{Q}$, ocurre un cambio que corresponde a una ganancia positiva de la cantidad de información y con ello se reduce la indeterminación, hecho que se sintetiza en la expresión siguiente (Volkenshtein, 1985):

$$
\Delta \mathrm{I}=\mathrm{K} \sum_{\mathrm{m}=1}^{\mathrm{n}} \mathrm{q}_{\mathrm{m}} \ln \left(\frac{\mathrm{q}_{\mathrm{m}}}{\mathrm{p}_{\mathrm{m}}}\right) \quad \mathrm{P} \rightarrow \mathrm{Q}
$$

De aquí se extrae una conclusión de relevancia: la información que recibe un sistema implica un aumento de la entropía del medio. Cualquier medición está ligada con el incremento de la entropía del mismo. Por tanto, el hecho de que la entropía informativa y la entropía termodinámica coincidan, se midan en unidades similares y en el mismo nivel de recepción, posibilita plantear cierta ley de conservación:

$$
\mathrm{I}+\mathrm{S}=\text { Constante }
$$

De la expresión (4) se infiere que:

$$
\Delta \mathrm{I}+\Delta \mathrm{S}=0, \quad \Delta \mathrm{S}=-\Delta \mathrm{I}
$$

De esta manera, si tiene lugar una disminución de la cantidad de información, la entropía aumenta y viceversa. Es así como la entropía constituye una medida de la insuficiencia de información sobre el sistema.

En un sistema, como por ejemplo en el caso más simple que consta de dos subsistemas con valores de entropías $S_{1}$ y $S_{2}$ respectivamente, si están dadas las distribuciones de probabilidades y la suma de las energías es constante, la entropía cumple con la propiedad de aditividad:

$$
\mathrm{S}=\mathrm{S}_{1}+\mathrm{S}_{2}
$$

Si el sistema no está equilibrado, las magnitudes que lo caracterizan, en particular, la entropía, constituyen funciones del tiempo, de manera que:

$$
\mathrm{S}(\mathrm{t})=-\mathrm{k} \sum_{\mathrm{m}=1}^{\mathrm{n}} \mathrm{p}_{\mathrm{i}}(\mathrm{t}) \ln \left(\mathrm{p}_{\mathrm{i}}(\mathrm{t})\right)
$$

En caso de que el sistema además se caracterice por un conjunto de variables extensivas, la razón de cambio de la entropía con el tiempo es igual a la suma de productos de fuerzas generalizadas y flujos generalizados. Dicha razón no es más que la función de disipación o velocidad de producción de entropía por unidad de volumen:

$$
\frac{\mathrm{d} S}{\mathrm{dt}}=\sum_{\mathrm{m}=1}^{\mathrm{n}} \mathcal{F}_{\mathrm{Gm}} \Phi_{\mathrm{m}}=\mathbb{F}_{\mathrm{D}}
$$

En los sistemas abiertos, el cambio de entropía consta de la variación generada en el interior $\left(\Delta \mathrm{S}_{\text {int }}\right)$ y del cambio de entropía procedente del medio externo o que se incorpora a este $\left(\Delta \mathrm{S}_{\mathrm{ext}}\right)$ :

$$
\Delta \mathrm{S}=\Delta \mathrm{S}_{\mathrm{int}}+\Delta \mathrm{S}_{\mathrm{ext}}
$$

Es de notar que $\Delta S_{\text {int }} \geq 0$ pues si se trata de un sistema aislado $\Delta \mathrm{S}_{\mathrm{ext}}=0$ y la entropía de tal sistema no puede disminuir. De la ecuación (9) se infiere la posibilidad de existencia de estados no equilibrados pero a la vez estacionarios. En tales estados las magnitudes termodinámicas que lo caracterizan son independientes del tiempo pero sus valores no están equilibrados. Por tanto la producción de entropía en el interior se compensa con la expulsión de entropía al medio ambiente, de modo que dichos estados se mantienen con el incremento de la entropía de dicho medio y que está determinado por el flujo entrópico que ingresa a él.

Un ensemble estadístico en no equilibrio contiene más información sobre el sistema que el ensemble en equilibrio, por lo que al primero le corresponde una entropía $\Delta \mathrm{S}_{\text {neq }}$ menor que al segundo $\Delta \mathrm{S}_{\text {eq }}$ : $\Delta S_{\text {neq }}-\Delta S_{\text {eq }} \leq 0$. Todo lo anterior permite afirmar que los sistemas abiertos se alimentan de entropía negativa o neguentropía que además puede considerarse una medida de la información posible sobre el sistema. Se concluye así que la transmisión de neguentropía en el sentido termodinámico estadístico se puede considerar como introducción de alguna información en el sentido cibernético 
(Terlietski, 1971). Tal valor negativo expresa la interrelación formal y semántica entre información y entropía física

\section{Discusión}

\section{Algunas implicaciones para la gestión de información}

La entropía constituye una propiedad esencial de un sistema con conductas probabilistas o ambiguas. $\mathrm{Su}$ empleo se ha difundido en otras ciencias, especialmente cuando se necesita una descripción de los estados de organización y desorganización estructural así como hacer referencia al grado de destrucción de las relaciones entre los elementos de un sistema (Osipov y Uvarov, 2004).

Las propiedades universales de la entropía la convierten en un recurso conceptual metodológico con un alcance que abarca más allá de las ideas que lo restringen al marco termodinámico. Dicho concepto puede extrapolarse a un conjunto de fenómenos que no necesariamente tienen que ver con procesos de naturaleza física. En éstos la propia entropía puede desempeñar el papel de parámetro para caracterizar el grado de desorden interno o de caos en sus estados.

Una variedad de tales fenómenos se da en sistemas sociales y sus subsistemas, aunque para estos el concepto de entropía aún no resulta claro. El mismo hace referencia a la complejidad sistémica como fenómeno emergente, cuyo marco teórico reside en las ideas de Bertalanffy y N. Wiener. La teoría de la entropía de sistema (SET) la conceptúa como indicador del estado interno del mismo y su desorden como variable temporal (Bailey, 2006). [16]. En un contexto más específico, la entropía social constituye una medida de la indeterminación y predictibilidad de un sistema. Si el estado futuro se considera altamente predecible, la entropía resulta baja y viceversa. Por tanto, un sistema de baja entropía se considera organizado y deseable (Mavrofides, Achilleas, Dimitris y Antonios, 2011), mientras que uno caracterizado por falta de organización o con una elevada variedad de estados posibles de realización, resulta altamente entropizado y poco deseable.

No obstante las distinciones hechas, las propiedades generales de la entropía pueden emplearse como herramientas en el aparato teórico para la descripción fenomenológica de sistemas en la sociedad que no son necesariamente sistemas físicos o biológicos. Así, los enfoques conceptuales y corolarios fundamentales tienen implicaciones esenciales al describirse el comportamiento de entidades, organizaciones o subsistemas en que la gestión de información constituye uno de los diversos procesos en los que se involucra.

Un conjunto rasgos posibilitan extrapolar el concepto de entropía a los subsistemas antes referidos aunque en ellos esta magnitud puede entenderse de diversas maneras y la aplicación no está exenta de interpretaciones no muy adecuadas.

Los sistemas típicamente abiertos y sus subsistemas en general, están constituidos por elementos que tienen acciones inherentes a entes disipativos. Estos se convierten en la principal fuente de información necesaria para el funcionamiento del sistema como un todo. Tales sistemas tienen un nivel de organización crítica. Si la regularidad del sistema es inferior a tal nivel, los procesos de incremento del orden prevalecen en él, pero si dicho nivel de regularidad está por encima, entonces los procesos que generan desorganización o chaos predominan. Durante la ocurrencia, si existe un equi-balance entre los mismos, el estado del sistema se torna estable (Markina y Dyachkov, 2014).

En los sistemas de tipo gaseoso, la entropía depende de la energía, el volumen y la cantidad de sustancia mientras que en los subsistemas sociales se vincula con el grado de desarrollo de las demandas de información y el nivel de incertidumbre informacional.

La entropía tiene una naturaleza aditiva lo cual está relacionado con el hecho de que el macroestado de un sistema depende de la cantidad probable de microestados. De aquí que la suma de la entropía de los elementos de un sistema es igual a la entropía del sistema en conjunto. Por otra parte en una entidad social y en particular en su sistema de información, se hace posible determinar recursos informacionales y por ende cuantificarla.

Otra particularidad de importancia consiste en que cualquier información adicional representa en la concepción entrópica, una contribución al aumento de la entropía negativa del sistema. De hecho, en entidades de la sociedad, el incremento del volumen de información no siempre conduce al decrecimiento de la entropía. Por ende, la entropía negativa de un sistema puede incrementarse sólo debido a la información de la calidad requerida y su entrada en ciertos instantes.

El aumento irreversible de la entropía solo tiene lugar en los sistemas cerrados, los cuales son simplemente aquellos que no interactúan con el medio externo. En los sistemas abiertos como lo representa cualquier subsistema de la sociedad, la entropía puede aumentar, permanecer constante o decrecer. Ello se debe a que en los mismos está 
presente la propia entropía del sistema que tiende a crecer constantemente y a la existencia de dos flujos entrópicos: el que llega desde el medio exterior y aquel en el que la entropía es cedida por el sistema a dicho medio o entorno (Markina y Dyachkov, 2014).

Es significativo también que la neguentropía ejerza un efecto de balance frente a la entropía propia del sistema de manera que su influencia equivale a ceder entropía al medio.

Cualquier suborganización o entidad social, al ser un sistema abierto se caracteriza por tasas de entropía cuya variación constituye una función de la cantidad y complejidad de las interrelaciones con el entorno. Ello permite explicar la transición que experimentan tales sistemas a estados de mayor entropía mientras mayor sea la cantidad de factores externos que influyan sobre él.

Uno de los más importantes desafíos de la modelación de los sistemas complejos socioeconómicos que tienen comportamientos estocásticos, consiste en hacer uso de las propiedades de la entropía. Es de especial interés utilizar la aditividad, con el fin de determinar los niveles y la calidad de la información necesarios para el buen funcionamiento de una organización así como para el análisis y determinación de la satisfacción de las demandas informacionales.

Una entidad social como puede ser una empresa, un centro de investigación o un departamento docente, por citar unos pocos ejemplos, no está aislada del resto de los subsistemas. Según Markina-Dyachkov (Markina y Dyachkov, 2014) un modelo de la gestión de una organización basado en la entropía, parte de considerar el medio externo contemplado el macroentorno y el microentorno, el medio interno, la entidad de gestión representada por los directivos que tienen la responsabilidad de tomar decisiones y el sistema de información organizacional.

Si la autoridad decisoria no tiene la posibilidad de determinar los factores influyentes en una situación en la que se involucra la entidad, en aras de adoptar las soluciones de gestión más efectivas, ello se traduce en una entropía incrementada del macroentorno y del microentorno. Tales circunstancias condicionan la entropía del medio interno.

La información necesaria para la toma de decisiones es adoptada a partir del vínculo con el sistema de información y en base a considerar el medio externo y el medio interno. Luego los datos requeridos proceden de diversas fuentes y del potencial informativo de quienes toman las decisiones. Es vital considerar que el sistema de información constituye un subsistema abierto y por ende, con un nivel de incertidumbre al que se asocia la entropía.

Así el cambio en la entropía de la entidad procede de la información que entra al sistema. De hecho tiene lugar una reducción de su entropía lo cual influye en el grado de satisfacción de las demandas de información. En consecuencia, disminuye la gama de alternativas de las decisiones administrativas a tomar.

Todo lo expresado constituye uno de los fundamentos del modelo referido (Markina $\mathrm{y}$ Dyachkov, 2014), en el que se establece una desigualdad con intervención de la entropía de la entidad y la entropía asociada al medio interno y al medio externo, dada por la siguiente expresión:

$$
\begin{aligned}
\Delta \mathrm{S}_{\text {ent }} \leq \Delta \mathrm{S}_{\text {int }}+ & \Delta \mathrm{S}_{\text {ext }} \\
& =\Delta \mathrm{S}_{\text {int }} \\
& +\left[\Delta \mathrm{S}_{\text {mic }}+\Delta \mathrm{S}_{\text {mac }}\right]
\end{aligned}
$$

donde:

$\Delta \mathrm{S}_{\text {int }}:$ entropía asociada al medio interno.

$\Delta \mathrm{S}_{\mathrm{ext}}=\Delta \mathrm{S}_{\mathrm{mic}}+\Delta \mathrm{S}_{\mathrm{mac}}$ : entropía asociada al medio externo.

$\Delta \mathrm{S}_{\text {mic }}$ : entropía del microentorno inmerso en el medio exterior.

$\Delta \mathrm{S}_{\mathrm{mac}}$ : entropía del macroentorno vinculado con el medio exterior.

La entropía negativa relacionada con el potencial informativo de los objetos de gestión se expresa en la habilidad de la entidad para el filtrado de los flujos informacionales entrantes al sistema y transformarlos en recursos de información. Estos consisten en la información que es recogida, procesada, seleccionada, acumulada y generada, sobre ciertos y sus rasgos. La gestión de neguentropía reduce indeterminaciones y proporciona orden.

En el curso de la toma de decisiones administrativas, la entidad de gestión propiamente dicha, ejecuta acciones determinadas que se encaminan a la reducción de las incertidumbres con relación a un objeto específico o a la organización como un todo. En estas condiciones se puede proponer una expresión para la entropía que es función de la cantidad de información y las habilidades, competencias y esfuerzos que despliega la entidad:

$$
\Delta S_{\text {ent }}=f(\Delta I)+f(\text { hce })
$$


La ecuación describe cualitativamente las interrelaciones entre la entropía de la entidad, el flujo informacional $y$ sus habilidades $y$ competencias del grupo gestor. La misma debe corresponder además al hecho de que la disminución de la entropía de la entidad se asocia al incremento del volumen de información y a la habilidad y competencia de la microentidad gestora.

Mientras tanto la entropía del sistema de información constituye una función de la entropía de la microentidad de gestión y de las entropías del medio externo e interno, de acuerdo a la expresión siguiente:

$$
\Delta S_{\text {inf }}=\mathcal{F}\left(\Delta S_{\text {ent }}, \Delta S_{\text {int }}, \Delta S_{\text {mic }}, \Delta S_{\text {mac }}\right)(11)
$$

El constante incremento de la demanda de información conduce a la necesidad de su gestión, acumulación y uso efectivo. Estos representan la condición que debe asegurarse para reducir la entropía organizacional. La microentidad debe con sus acciones reducir las incertidumbres del sistema en conjunto, generar una variedad de estados posibles u ofrecer una gama más completa de todos los estados posibles del sistema determinada por las acciones del medio externo o la influencia de factores internos y que hagan tender el sistema hacia el estado deseado menos entrópico, más ordenado.

\section{Conclusiones}

A pesar de la distinción entre el significado de entropía en la termodinámica y entropía en los subsistemas sociales, la misma constituye una medida de la incertidumbre en un contexto social lo cual indica su carácter de entropía estadística no desligado de la concepción termodinámica. Ello la convierte en un recurso metodológico para enfocar la gestión de información en las entidades en las cuales dicho proceso resulta esencial y así propiciar más orden, menos incertidumbres y comportamientos poco caotizados durante el funcionamiento de la organización como un todo.

\section{Bibliografía}

Ashby RW. (1957). An Introduction to Cybernetics. London: Chapman \& Hall Ltd.

Bailey, K.D. (2006). Living systems theory and social entropy theory. Systems Research and Behavioral Science 23, pp: 291-300.

Bustelo Ruesta, C. y Amarilla Iglesias, R. Gestión del conocimiento y gestión de la información. (2001). Boletín del Instituto Andaluz de Patrimonio Histórico, año VIII, no. 34, marzo 2001; pág. 226- 230.
Castells, M. (2000) The Information Age: economics, society and culture. Moscow: State University, Higher School of Economics; pp: 452.

Dmitriev, V.I. (1991). Teoría de la Información Aplicada. Moscú: Editorial Mir.

Hartley, R.V.L. (1928). Transmission of Information. Bell System Technical Journal, 7, 3, pp: 535-563.

Jae-Yoon, J.; Chang-Ho, C. and Cardoso, J. (2011). An entropy-based uncertainty measure of process models. Journal Information Processing Letters, Volume 111, Issue 3, January, 2011, pp: 135-141.

Markina, I.; Dyachkov, D. (2014). Entropy Model Management of Organization. World Applied Sciences Journal 30 (Management, Economics, Technology \& Tourism): pp: 159-164.

Mavrofides, T.; Achilleas, K.; Dimitris, P.; Antonios, L. (2011). On the Entropy of Social Systems: A Revision of the Concepts of Entropy and Energy in the Social Context. Systems Research and Behavioral Science. Published online in Wiley Online Library. $\quad$ DOI: 10.1002/sres.1084 (wileyonlinelibrary.com)

Mihail, N.N.; Mircea, A.R. (1989). ¿Qué es la Biocibernética? La Habana: Editorial Científico-Técnica.

Osipov, A.I.; Uvarov, A.V. (2004). Entropy and its role in science. Soros Educational Journal, 8(1), pp: 70-79.

Прангишвили И. В. (2003). Энтропийные и другие системные закономерности : Вопросы управления сложными системами. Москва: Наука; с. 149-150.

Schwarz, M. (2014). Una medida de la incertidumbre basada en la entropía para cuantificar el impacto de la pérdida de información en los Sistemas de Gestión. Revista Escuela de Posgrado Paideia XXI 4 (5), pág. 46-56.

Song, Z.; Liang, P. and Ni, L. (2015). Order Degree Evaluation of Information System Based on Improved Structural Entropy. Open Journal of Business and Management, 3, pp: 235-242. 
Shannon, C. (1948). A Mathematical Theory of Communication. The Bell System Technical Journal, Vol. 27, pp. 379-423, 623-656, July, October.

Terlietski, Y.P. (1971). Física Estadística. La Habana: Ediciones de Ciencia y Técnica, Instituto Cubano del Libro.

Volkenshtein, M.V. (1985). Biofísica. Moscú: Editorial Mir.

Wiener, N. (1965). Cybernetics or the Control and Communication in the Animal and the Machine. Massachusett: MIT Press. 